\title{
COMPOSIÇÃO QUÍMICA E ATIVIDADE ANTICOLINESTERÁSICA DE UMA FRAÇÃO ATIVA DO EXTRATO DE FOLHAS DE Citrus limon (L.) Burm
}

Rusbene Bruno Fonseca de Carvalho*, Antonia Amanda Cardoso de Almeida e Rivelilson Mendes de Freitas

Rede Nordeste de Biotecnologia, Universidade Federal do Piauí, Campus Ministro Petrônio Portela, 64049-550 Teresina - PI, Brasil Luciano Silva Lima

Instituto Federal da Bahia, Campus Porto Seguro, 45810-000 Porto Seguro - BA, Brasil

Juceni Pereira David

Faculdade de Farmácia, Universidade Federal da Bahia, Campus de Ondina, 40170-290 Salvador - BA, Brasil

Jorge Mauricio David

Instituto de Química, Universidade Federal da Bahia, Campus de Ondina, 40170-290 Salvador - BA, Brasil

Chistiane Mendes Feitosa

Departamento de Química, Centro de Ciências da Natureza, Universidade Federal do Piauí, Campus Ministro Petrônio Portela, 64049-550 Teresina - PI, Brasil

Recebido em 18/4/13; aceito em 9/6/13; publicado na web em 2/8/13

\begin{abstract}
CHEMICAL COMPOSITION AND ANTICHOLINESTERASE ACTIVITY OF AN ACTIVE FRACTION OF EXTRACT OF Citrus limon (L.) Burm LEAVES. This paper describes the chemical composition and acetylcholinesterase inhibition of an active chromatographic fraction of the EtOAc extract of Citrus limon leaves. The composition of the active fraction presented a mixture of two coumarins, 5,8-dimethoxy-psoralen and 5,7- dimethoxycoumarin, identified by ${ }^{1} \mathrm{H}$ and ${ }^{13} \mathrm{C}$ NMR data analysis, including DEPT, COSY, HMBC and HSQC experiments. It was also demonstrated that this mixture presents qualitative and quantitative AChE inhibition. In vitro studies indicated a CE50 value of $340 \mu \mathrm{g} / \mathrm{mL}$ with $95 \%$ of confidence. In vivo studies (10 and $25 \mathrm{mg} / \mathrm{kg}$ ) revealed inhibition of 30.09 and $30.06 \%$ of AChE activity in relation to neostigmine, respectively.
\end{abstract}

Keywords: Citrus limon (L.) Burm; psoraleno; acetylcholinesterase.

\section{INTRODUÇÃO}

As plantas têm sido utilizadas com fins medicinais desde o inicio da civilização humana com a finalidade de prevenção, tratamento e cura de diversas doenças. ${ }^{1,2}$ Nas práticas tradicionais da medicina chinesa, diversas plantas têm sido usadas para tratar distúrbios cognitivos, incluindo doenças neurodegenerativas como a doença de Alzheimer (DA). ${ }^{3}$ A DA é caracterizada por um panorama progressivo de demência comprometendo inicialmente a memória para fatos recentes. Posteriormente, com a evolução da doença pode haver a deteriorização das funções cognitivas com apraxias construtivas, agnosias e distúrbios afásicos. ${ }^{4}$

Dados estatísticos apontam que tal doença acomete aproximadamente 5 a $10 \%$ da população com idade superior a 65 anos e 20 a $40 \%$ daqueles com faixa etária superior a 85 anos. ${ }^{5}$ É estimado que em 2050 , mais de $25 \%$ da população mundial será idosa, sugerindo um aumento da prevalência e da incidência desta doença, sendo, portanto, extremamente necessária a busca por novas formas de tratamento mais eficazes e seguras..$^{6-8}$

As áreas cerebrais patologicamente mais afetadas na DA é o hipocampo e o neocórtex. Estas áreas estão associadas às funções mentais de forma mais predominante. Um promissor tratamento para doença é o aumento do nível de acetilcolina no cérebro usando inibidores da acetilcolinesterase (AChE), justificando as pesquisas por novos agentes mais eficazes e com menor custo para a saúde humana. ${ }^{9,10}$

Os portadores de DA têm níveis reduzidos de acetilcolina, neurotransmissor que intervém nos processos da memória. ${ }^{10}$ Inibidores de AChE são amplamente utilizados no tratamento, uma vez que estes alteram a função colinérgica central ao inibir as enzimas que

*e-mail: rusbenebruno@hotmail.com degradam a acetilcolina (enzimas acetilcolinesterase e butirilcolinesterase), ampliando a capacidade da acetilcolina de estimular os receptores cerebrais, nicotínicos e muscarínicos. ${ }^{10,11}$

Desde a introdução de medicamentos como Tacrina, Donepezil, Rivastigmina e Galantamina na prática clínica, os inibidores da AChE constituem o tratamento sintomático de escolha para a DA, porém alguns ainda apresentam efeitos colaterais indesejados sobre os diversos órgãos e sistemas, como, sistema cardiovascular, sistema respiratório, trato gastrintestinal, bexiga e sistema nervoso central. ${ }^{7,12}$

Nesse contexto e após realizar uma revisão de literatura, podem ser apontadas diversos trabalhos com extratos de plantas medicinais e/ou de compostos isolados do gênero Citrus, como adstringente, antioxidante, antianêmico, antibiótico, antisséptico, antiemético, antidepressivo, anti-inflamatório, antiespasmódico, bactericida, antireumático e antidisentérico. ${ }^{2,10,13,14}$ Muitas destas atividades podem ser relevantes para o tratamento da DA, justificando a seleção de $C$. limon utilizada nesse estudo para essa possível aplicação terapêutica.

A família Rutaceae consiste aproximadamente de 150 gêneros e 1.600 espécies, distribuídas amplamente em regiões tropicais, subtropicais e temperadas do mundo. ${ }^{15}$ No Brasil, a família está representada por aproximadamente 29 gêneros e 182 espécies, com algumas de importância medicinal, ecológica e econômica. ${ }^{16}$ O Citrus é um gênero que compreende aproximadamente 70 espécies que são ricas em flavonóides, óleos voláteis, cumarinas e pectinas cerebrais. ${ }^{10}$

Citrus limon (Rutaceae) é uma planta do norte e nordeste do Brasil, conhecida popularmente como "Limoeiro", cujas folhas e frutos são aproveitados pela medicina popular para diversos fins terapêuticos. ${ }^{10,17}$ Nessa perspectiva, o presente estudo teve como objetivo isolar e identificar compostos com potencial inibição da enzima acetilcolinesterase, bem como realizar uma avaliação 
qualitativa e quantitativa da atividade desses compostos para verificar suas propriedades farmacologicas com ênfase na prevenção e/ ou tratamento da DA.

\section{PARTE EXPERIMENTAL}

\section{Material vegetal e procedimento para obtenção dos extratos}

As folhas da espécie C. limon (L.) Burm foram coletadas às 8 horas da manhã durante o mês de Fevereiro de 2010 de hortas medicinais existentes nas proximidades do Campus Senador Helvídio Nunes de Barros da Universidade Federal do Piauí no município de Picos, Piauí. Identificada e depositada no Herbário Graziela Barroso da Universidade Federal do Piauí (UFPI), recebendo a exsicata o número 26.453.

Os extratos foram preparados partindo-se de $1000 \mathrm{~g}$ de folhas de C. limon (L.) Burm que foram secas à temperatura ambiente e maceradas estaticamente. O material obtido (300 g) foi submetido à extração por um período de cinco dias com os solventes hexano, acetato de etila (AcOEt) e metanol $(\mathrm{MeOH})$, respectivamente, sendo em seguida concentrados por evaporador rotatório sob pressão reduzida, obtendo-se então os seguintes extratos brutos e seus respectivos rendimentos: hexânico $(8,5 \mathrm{~g} / 2,85 \%)$, acetato de etila $(9,6 \mathrm{~g} / 3,2 \%)$ e metanólico (28 g / 9,3\%). Estes foram cromatografados separadamente em coluna de sílica gel e monitoradas por cromatografia em camada delgada.

\section{Isolamento dos constituintes do extrato de acetato de etila da C. limon (L.) Burm}

O extrato AcOEt (9,62 g) foi submetido à separação por coluna cromatográfica sob gel de sílica 60, utilizando-se como eluentes, gradiente de Hexano/DCM, DCM/AcOEt, AcOEt/MeOH e MeOH puro, obtendo desse processo 18 frações (F 1-18). As frações DCM/ AcOEt (7:3, F 10-11) 1,2215 g foram refracionadas em CC de gel de sílica, utilizando os eluentes DCM , DCM/ AcOEt, AcOEt, AcOEt/ $\mathrm{MeOH}$ e $\mathrm{MeOH}$, gerando 14 frações (F 10-11/1-14).

As frações DCM/AcOEt (F 2-10) $297 \mathrm{mg}$ foram reagrupadas e resubmetidas a cromatografia utilizando os eluentes hexano, hexano/ DCM, DCM, DCM/AcOEt, AcOEt, AcOEt/MeOH e MeOH, gerando 62 frações (F 2-10/1-62). As frações DCM (F 18-37) $99 \mathrm{mg}$ foram reagrupadas e submetidas a novo tratamento cromatográfico com hexano, DCM, DCM/AcOEt , AcOEt e MeOH, tendo por fim 24 frações (F 18-37/1-24). As sub-frações (F 2-14) constituída de um pó branco (20 mg), solúvel em diclorometano, com a faixa de ponto de fusão $91-92{ }^{\circ} \mathrm{C}$; o valor do TLC em clorofórmio $100 \%, \mathrm{Rf}=0,48$, que embora apresentasse uma única mancha em CCD, revelou tratar-se de uma mistura de dois constituintes.

A mistura isolada foi caracterizada através da análise de dados obtidos pelos espectros de RMN de ${ }^{1} \mathrm{H}$, RMN de ${ }^{13} \mathrm{C}$, DEPT 135 , COSY, HMBC e HSQC além de comparação com dados da literatura. Os espectros de RMN ${ }^{1} \mathrm{H}$ e ${ }^{13} \mathrm{C}$ foram registrados em espectrômetro da Varian, modelo Gemini 2000, operando a $300 \mathrm{MHz}$ para o ${ }^{1} \mathrm{H} \mathrm{e}$ $75 \mathrm{MHz}$ para ${ }^{13} \mathrm{C}$. Os deslocamentos químicos foram registrados em $\delta(\mathrm{ppm})$ em referência ao TMS.

\section{Ensaio em cromatografia de camada delgada (CCD) - Atividade de inibição qualitativa}

A amostra contendo a mistura dos constituintes isolados foi dissolvida em metanol para uma concentração de $1 \mathrm{mg} / \mathrm{mL}$, em seguida aplicada em CCD (DC Alufolien, Silicagel $60 \mathrm{~F}_{254}, 0,2 \mathrm{~mm}$, Merck), e eluída em clorofórmio:metanol 9:1. Após a placa ser desenvolvida, a atividade inibitória foi detectada utilizando revelador baseado no método de Ellman ${ }^{18}$ modificado por Rhee e colaboradores. ${ }^{19}$

A placa foi pulverizada com DTNB (ácido 5,5'-ditiobis-[2-nitrobenzóico)/ATCI (Iodeto de acetiltiocolina) (1 mM DTNB e 1 mM ATCI em tampão A). Depois de seca (5 min) foi pulverizada com 3 units/mL da enzima acetilcolinesterase, tipo VI-s, liofilizada, 292 U/mg sólida, 394 U/mg proteína (Sigma Chemical Co.) e, após 10 min, foi observada a coloração amarela. A visualização da inibição foi feita pela observação de halos brancos. A coloração desaparece em aproximadamente 15 a $30 \mathrm{~min}$. A cafeína foi utilizada como substancia padrão. ${ }^{20}$

\section{Avaliação in vitro da atividade da enzima acetilcolinesterase}

O efeito inibitório da mistura dos constituintes isolados sobre atividade da enzima acetilcolinesterase in vitro é avaliada por uma adaptação do método espectrofotométrico de Ellman e colabordores ${ }^{18}$ e os procedimentos para o ensaio foram detalhados posteriormente em estudos realizados por Moyo e colaboradores. ${ }^{21}$

Para a atividade de inibição quantitativa, foi utilizado um espectrofotômetro Biospectro System 220. Inicialmente, $100 \mu \mathrm{L}$ da amostra (concentrações de 0,1, 0,05, 0,025, 0,0125 e 0,00625 mg/ $\mathrm{mL}$ em solução tampão $50 \mathrm{mM}$ Tris- $\mathrm{HCl}, \mathrm{pH}$ 8, e $10 \%$ de metanol) foram misturados com $100 \mu \mathrm{L}$ de $\operatorname{AChE}(0,22 \mathrm{U} / \mathrm{mL}$ em $50 \mathrm{mMol} / \mathrm{L}$ Tris-HCl, tampão pH 8 , 0,1\% BSA) e $200 \mu \mathrm{L}$ de tampão ( 50 mMol/L Tris- $\mathrm{HCl}, \mathrm{pH} 8,0,1 \%$ BSA). A mistura foi incubada durante $5 \mathrm{~min}$ a $30{ }^{\circ} \mathrm{C}$. Posteriormente, $500 \mu \mathrm{L}$ de ácido ditiobisnitrobenzóico (DTNB) ( $3 \mathrm{mMol} / \mathrm{L}$ em Tris-HCl, pH 8, 0,1 mol/L de NaCl, 0,02 mol/ $/ \mathrm{MgCl}_{2}$ ) e $100 \mu \mathrm{L}$ de iodeto de acetiotiocolina (ATCI) ( $4 \mathrm{mMol} / \mathrm{L}$ em água) foram adicionados.

Um teste branco também foi preparado substituindo a enzima AChE com $100 \mu \mathrm{L}$ de tampão (50 mMol/L Tris- $\mathrm{HCl}, \mathrm{pH} 8,0,1 \%$ BSA). As amostras foram analisadas em triplicata. A reação foi monitorada durante 5 min em $412 \mathrm{~nm}$ e velocidade inicial $\left(\mathrm{V}_{0}\right)$ gravada. Como controle negativo foi utilizado o Tampão $(0,1 \%$ de metanol em $50 \mathrm{mMol} / \mathrm{L}$ Tris-HCl, pH 8, 10\%) e o medicamento neostigmina (Atrovent ${ }^{\circledR}$ ) foi usado como padrão. A atividade anticolinesterásica (\%I) foi calculada de acordo com a seguinte equação:

$$
\mathrm{I}(\%)=1-\left(\frac{\text { Vo amostra }}{\text { Vo branco }}\right) \times 100
$$

Vo amostra e Vo branco representam as velocidades iniciais de amostras e branco. Os valores da concentração efetiva inibitória de $50 \%\left(\mathrm{CE}_{50}\right)$ foram obtidos por intermédio de plotagem Log-Probit.

\section{Avaliação do efeito inibitório da mistura dos constituintes isolados do extrato de acetato de etila das folhas de $C$. limon (L.) Burm na atividade da enzima acetilcolinesterase in vivo}

\section{Animais}

Nesse estudo foram utilizados 32 camundongos Swiss machos albinos (Mus musculus) adultos com dois meses de idade com peso variando entre 25 e 30 gramas, provenientes do Biotério Central do Departamento de Ciências Agrárias da Universidade Federal do Piauí.

Os animais foram divididos em quatro grupos de oito camundongos para a avaliação do efeito inibitório da fração isolada na atividade da enzima acetilcolinesterase. O primeiro grupo foi tratado com Tween $800,05 \%$ dissolvido em solução salina $0,9 \%$ por via oral (v.o.) (controle negativo; $n=8$ ), o grupo controle positivo foi tratado com neostigmina ( $25 \mathrm{mg} / \mathrm{kg}$; via intraperitoneal (i.p.); $n=8)$ e os dois outros grupos foram tratados com a mistura dos compostos isolados nas doses de 10, $25 \mathrm{mg} / \mathrm{kg}$ (v.o.; $n=8)$. 
Após os tratamentos, os animais foram colocados em gaiolas de acrílico $\left(30\right.$ x $\left.30 \mathrm{~cm}^{2}\right)$ com água e ração ad libitum e observados durante 24 horas para verificação dos parâmetros comportamentais. Durante esse período foi registrado o número de morte entre os animais de cada grupo, os animais que sobreviveram ao período de observação foram anestesiados pela administração de pentobarbital sódico (10-15 mg/100 g de peso, i.p.) e em seguida, eutanasiados por decapitação para a remoção do encéfalo e dissecação da área cerebral (hipocampo) em ambos os lados dos cérebros em estudo.

A atividade enzimática de acetilcolinesterase no hipocampo de camundongos foi medida por meio de métodos espectrofotométricos e os resultados comparados aos valores obtidos dos grupos tratados com o veículo (controle negativo; $n=8$ ) e com neostigmina (controle positivo; $n=8)$.

O experimento foi realizado de acordo com o guia de cuidados e usos de animais de laboratório do Departamento de Saúde e Serviços Humanos dos Estados Unidos da América (EUA). Todos os experimentos desenvolvidos neste estudo foram aprovados pelo Comitê de Ética em Experimentação com Animais da UFPI (CEEA / UFPI \# 44/09). Os procedimentos referentes à eutanásia dos animais estavam em conformidade com o Parágrafo Único do Artigo $2^{\circ}$ da Resolução N $^{\circ} 714$, de 20 de Junho de 2002 do Conselho Federal de Medicina Veterinária - CFMV

\section{Avaliação da atividade da enzima acetilcolinesterase in vivo}

De acordo com método descrito por Ellman ${ }^{18}$ foi determinada a atividade acetilcolinesterásica, utilizando como princípio básico a medida da velocidade de produção de tiocolina a partir do substrato acetiltiocolina (ATC), a proporção em que a ATC era hidrolisada.

Os tecidos hipocampais foram homogeneizados em tampão fosfato ( $\mathrm{pH} 8,0 ; 0,1 \mathrm{M}) 10 \%$ e o homogenato $(10 \mu \mathrm{L})$ foi adicionado a uma cubeta contendo $500 \mu \mathrm{L}$ do tampão, $890 \mu \mathrm{L}$ de água destilada e $50 \mu \mathrm{L}$ de ácido ditiobisnitrobenzóico (DTNB) 0,01M e a absorbância zerada. Após a absorbância ser deixada em zero, a cubeta foi retirada e a ela foi acrescentado iodeto de acetiotiocolina 0,075 M. A absorbância foi registrada durante $3 \mathrm{~min}$ em $412 \mathrm{nM}$. A atividade da enzima foi calculada como modificações na absorbância do minuto 3 para o minuto 0 , relativo ao conteúdo de proteína contido no homogenato. ${ }^{22} \mathrm{O}$ procedimento completo foi feito usando um espectrofotômetro Biospectro SP 220 ajustado para um comprimento de onda de $412 \mathrm{nM}$.

\section{RESULTADOS E DISCUSSÃO}

\section{Identificação da mistura dos constituintes isolados do extrato de acetato de etila das folhas de $C$. limon}

A análise dos espectros de RMN de ${ }^{1} \mathrm{H}$ e de ${ }^{13} \mathrm{C}$, incluindo experimentos de DEPT, permitiu concluir que a fração é formada por uma mistura de duas cumarinas principais. Pode ser verificado no espectro de $\mathrm{RMN}{ }^{1} \mathrm{H}$ a existência uma substância predominante. Pela análise das integrais pode ser concluído que aproximadamente $85 \%$ da fração é composta de uma furanocumarina do tipo linear.

Os espectros bidimensionais HMQC e HMBC permitiram correlacionar os dados observados de hidrogênio com os carbonos diretamente ligados e vizinhos, facilitando a identificação do componente principal. Os dados de RMN do composto principal foram comparados com os descritos na literatura, ${ }^{23}$ permitindo assim identificar de maneira inequívoca a presença do 5,8-dimetoxipsoraleno (A) (Figura 1) como sendo o componente principal.

5,8-Dimetoxipsoraleno (A) RMN de ${ }^{1} \mathrm{H} \mathrm{NMR}\left(300 \mathrm{MHz}, \mathrm{CDCl}_{3}\right)$ : d (ppm): $4.17\left(6 \mathrm{H}, \mathrm{s}, 2 \times \mathrm{OCH}_{3}\right), 6.29(1 \mathrm{H}, d, J=10 \mathrm{~Hz}, \mathrm{H}-3), 7.0$ $(1 \mathrm{H}, d, J=2.4 \mathrm{~Hz}, \mathrm{H}-9), 7.63(1 \mathrm{H}, d, J=2.8 \mathrm{~Hz}, \mathrm{H}-10), 8.13(1 \mathrm{H}$, $d, J=9.6 \mathrm{~Hz}, \mathrm{H}-4)$. RMN de ${ }^{13} \mathrm{C}\left(75 \mathrm{MHz}, \mathrm{CDCl}_{3}\right) \mathrm{d}(\mathrm{ppm}): 160.42$ (C-2); 112,77 (C-3); 139,41 (C-4); 107,54 (C-4a); 144,25 (C-5); 114,72 (C-6); 149,95 (C-7); 144,25 (C-8), 138,73 (C-8a); 105,07 (C-9); 145,10 (C-10); 60,71 (5- $\left.\mathrm{OCH}_{3}\right) ; 60,71\left(8-\mathrm{OCH}_{3}\right)$.

Análise dos sinais de RMN da substância presente em menor quantidade na fração permitiu sugerir a presença de uma cumarina dimetoxilada. Os sinais observados no espectro de RMN de ${ }^{13} \mathrm{C}$ em d 94,7 e 92,7 foram indicativos que a segunda substância era uma cumarina substituída nas posições 5 e 7. Além disso, os dados de RMN dos grupos metoxílicos corroboram com a proposta que o composto em menor quantidade como sendo a 5,7-dimetoxicumarina (B) (Figura 1).

5,7-Dimetoxicumarina (B) RMN de ${ }^{1} \mathrm{H} N M R\left(300 \mathrm{MHz}, \mathrm{CDCl}_{3}\right)$ : d (ppm): 3,82 (3H, s, $\left.\mathrm{OCH}_{3}-5\right), 3,86\left(3 \mathrm{H}, \mathrm{s}, \mathrm{OCH}_{3}-7\right), 6.23(1 \mathrm{H}, d, J$ $=$ indt, $\mathrm{H}-3), 6,18(1 \mathrm{H}, d, J=1,9 \mathrm{~Hz}, \mathrm{H}-6), 6,20(1 \mathrm{H}, d, J=1,9 \mathrm{~Hz}$, $\mathrm{H}-8) 7,93(1 \mathrm{H}, d, J=10,2 \mathrm{~Hz}, \mathrm{H}-4)$. RMN de ${ }^{13} \mathrm{C}\left(75 \mathrm{MHz}, \mathrm{CDCl}_{3}\right)$ d (ppm): 162.30 (C-2); 110,87 (C-3); 138,73 (C-4); 106,69 (C-4a); 143,61 (C-5); 94,78 (C-6); 156,91 (C-7); 92,73 (C-8). C8a não foi detectado.

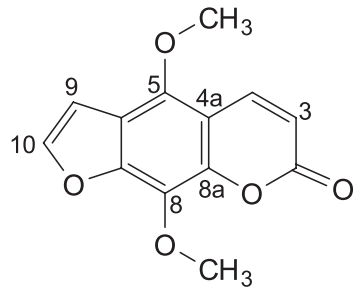

(A)<smiles>COc1cc(OC)c2ccc(=O)oc2c1</smiles>

(B)
Figura 1. Estrutura química da mistura dos constituintes isolados do extrato de acetato de etila das folhas de C. limon (L.) Burm

Avaliação da inibição qualitativa da mistura dos constituintes isolados do extrato de acetato de etila das folhas de Citrus limon (L.) Burm

A amostra contendo a mistura dos constituintes isolados da $C$. limon (L.) Burm apresentou resultado positivo frente à inibição qualitativa da enzima acetilcolinesterase. $\mathrm{O}$ resultado foi observado através da placa de CCD que apresentou coloração amarela, com halos brancos, sugerindo que a fração possui ação inibitória sobre a enzima de AChE. A constatação da inibição é possível seguindo-se a metodologia de Elmann, ${ }^{18}$ adaptada por Rhee e colaboradores. ${ }^{19}$ Comparando aos resultados de outros estudos, nossos dados corroboram com a hipótese de que compostos cumarínicos apresentam um potencial anticolinesterásico. ${ }^{8,24}$

\section{Avaliação do efeito inibitório da mistura dos constituintes isolados do extrato de acetato de etila das folhas de $C$. limon (L.) Burm na atividade da enzima acetilcolinesterase in vitro}

Em nossos estudos in vitro foi verificado uma inibição da atividade da AChE de 95,9\% quando a neostigmina (controle positivo) foi usada em uma concentração de $0,1 \mathrm{mg} / \mathrm{mL}$. Quando avaliada a mistura dos constituintes isolados do extrato AcOET das folhas de C. limon (L.) Burm nas concentrações de 0,1, 0,05, 0,025, 0,0125 e $0,00625 \mathrm{mg} / \mathrm{mL}$ foi detectado uma inibição de 57,75, 49,89, 35,03, 23,78 e $8,71 \%$ na atividade da AChE in vitro, respectivamente. Com base nesses resultados também foi determinada a $\mathrm{CE}_{50}$ que corresponde a $0,061 \mathrm{mg} / \mathrm{mL}$ com intervalo de confiança de $95 \%(0,033$ a $\left.0,18 \mathrm{mg} / \mathrm{mL} ; \mathrm{r}^{2}=0,9835\right)$.

Estudos com óleos essenciais e extratos já foram divulgados na literatura com atividade anticolinesterásica in vitro. As espécies 
C. sinensis (L) Osbeck, ${ }^{13}$ S. lavandulaefolia Vahl.,${ }^{25}$ Eucalyptus camaldulensis Dehnhardt e Ocimum canum Sims $^{26}$ apresentaram um $\mathrm{CE}_{50}$ da atividade de AChE igual a 0,063, 0,05, 0,018, 0,036 mg/mL, respectivamente. Os extratos em éter de petróleo, diclorometano e metanol das folhas da Leucosidea sericea Eckl. \& Zeyh. ${ }^{27}$ apresentam $\mathrm{CE}_{50}$ de 0,$16 ; 0,14$ e $0,24 \mathrm{mg} / \mathrm{mL}$, respectivamente.

Nessa perspectiva, a mistura dos constituintes isolados do extrato de AcOEt das folhas de C. limon (L.) Burm (5,8-dimetoxi-psoraleno e 5,7-dimetoxicumarina) apresentou um valor para $\mathrm{CE}_{50}$ menor que os valores mensurados para os extratos obtidos de outras espécies de plantas medicinais já estudados. No entanto, quando comparado o valor inibitório da atividade da $\mathrm{AChE}$ correspondente a $\mathrm{CE}_{50}$ determinada em nosso estudo foi verificado que essa $\mathrm{CE}_{50}$ foi inferior apenas em comparação ao valor detectado para o óleo essencial extraído das folhas de $C$. sinensis (L) Osbeck. ${ }^{13}$

Dessa forma, nosso estudo sugere que a referida fração pode demonstrar resultados inibitórios sobre a atividade da $\mathrm{AChE}$ in vitro, com potencial aplicação em modelos farmacológicos experimentais em roedores para avaliar seu potencial farmacológico em ensaios pré-clínicos em procedimentos experimentais que simulem as patologias relacionadas com o sistema nervoso central, nas quais a modulação da $\mathrm{AChE}$ responda pela fisiologia inerente dos transtornos psicossociais, particularmente a DA.

\section{Avaliação do efeito inibitório da mistura dos constituintes isolados do extrato de acetato de etila das folhas de $C$. limon (L.) Burm na atividade da enzima acetilcolinesterase in vivo}

Complementando a analise dos efeitos inibitórios sobre a atividade de AChE, nesse estudo foi observado que no grupo $(n=8)$ tratado com neostigmina (droga de referência) apenas cinco animais sobreviveram ao período de observação, no qual pode ser verificado uma redução de $43,32 \%$ na atividade da enzima acetilcolinesterase quando comparado ao grupo controle negativo $[10,03 \pm 0,16$; $\mathrm{q}=6,060 ; \mathrm{p}<0,001]$.

Por sua vez, o tratamento com 10 e $25 \mathrm{mg} / \mathrm{kg}$ da referida fração isolada do extrato AcOEt das folhas de $C$. limon (L.) Burm reduziu em $60,37 \%[3,974 \pm 0,66 ; q=6,06 ; p<0,001]$ e $60,36 \%[3,976 \pm$ $0,43 ; \mathrm{q}=6,05 ; \mathrm{p}<0,001]$ a atividade da AChE em relação ao controle negativo $[10,03 \pm 0,16]$, respectivamente. Além disso, em comparação ao grupo controle positivo tratado com neostigmina $[5,685 \pm 0,47]$ foi detectada uma redução de 30,09 e 30,06\% na atividade da AChE entre os camundongos tratados com a dose de $10 \mathrm{mg} / \mathrm{kg}$ [3,974 $\pm 0,66$; $\mathrm{q}=1,711 ; \mathrm{p}<0,001]$ e $25 \mathrm{mg} / \mathrm{kg}[3,976 \pm 0,43 ; \mathrm{q}=1,709 ; \mathrm{p}<0,001] \mathrm{da}$ referida fração isolada de $C$. limon (L.) Burm .

A referida fração isolada de $C$. limon (L.) Burm não produziu alteração na atividade da $\mathrm{AChE}$ entre os camundongos tratados com a dose de $10 \mathrm{mg} / \mathrm{kg}[3,974 \pm 0,66]$ em relação ao grupo tratado com a dose de $25 \mathrm{mg} / \mathrm{kg}$ [3,976 $\pm 0,43 ; \mathrm{p}>0,05]$ (Figura 2). Sugerindo que o referido composto pode ser usado em menor dose, bem como pode aumentar de forma mais eficaz a estimulação colinérgica que a droga de referência.

Dessa forma, os dados fornecem indícios para a obtenção de composto com melhor aplicabilidade em estudos farmacológicos para o tratamento e/ou profilaxia do quadro de demência relacionado com alterações na atividade da enzima acetilcolinesterase. Nossos dados também revelam que a referida fração pode não apresentar propriedades tóxicas em ensaios pré-clinicos, uma vez que nenhum dos animais tratados com a doses de 10 e $25 \mathrm{mg} / \mathrm{kg}$ morreram durante o período de observação de 24 horas, entretanto, os camundongos tratados com a dose de $25 \mathrm{mg} / \mathrm{kg}$ da neostigmina apresentaram uma taxa de mortalidade de $37,5 \%$ durante o mesmo período de observação.

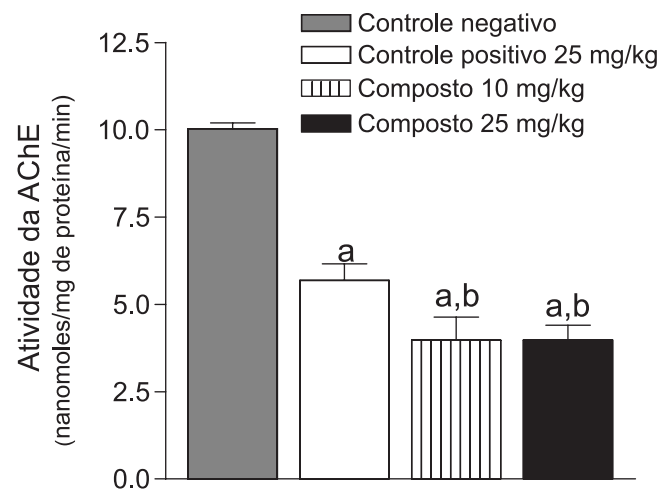

Figura 2 - Efeito da mistura dos constituintes isolados do extrato de acetato de etila das folhas de Citrus limon (L.) Burm sobre a atividade da enzima acetilcolinesterase (AChE) no hipocampo de camundongos. Os grupos foram tratados conforme descritos no protocolo experimental ( $n=8$ por grupo). Os valores representam a média \pm E.P.M. do número de animais usados nos experimentos. A atividade da acetilcolinesterase foi determinada em $10 \mathrm{~mL}$ de homogenato. Para análise estatística foram usados ANOVA e teste t-Student-Neuman-Keuls como post-hoc test. ${ }^{a}$ quando comparado ao controle negativo ( solução salina 0,9\%; $p<0,05$ ); ${ }^{b}$ quando comparado ao controle positivo (neostigmina $25 \mathrm{mg} / \mathrm{kg} ; \mathrm{p}>0,05)$. Composto $=$ mistura dos constituintes isolados do extrato de acetato de etila das folhas de C. limon (L.) Burm

\section{CONCLUSÕES}

Nossos estudos sugerem que a fração isolada da C. limon (L.) Burm pode demonstrar resultados inibitórios sobre a atividade da AChE in vitro e in vitro, com potencial aplicação em doenças neurodegenerativas que dependem da modulação desta enzima, incluindo a DA. A amostra contendo a mistura dos constituintes isolados não apresentou propriedades tóxicas em roedores, uma vez que nenhum dos animais tratados com a doses de 10 e $25 \mathrm{mg} / \mathrm{kg}$ morreram durante o período de observação de 24 horas. Sugerindo que o referido composto pode ser usado em menor dose, bem como pode aumentar de forma mais eficaz a estimulação colinérgica.

\section{AGRADECIMENTOS}

Ao Conselho Nacional de Desenvolvimento Científico e Tecnológico (CNPq) e à Fundação de Amparo à Pesquisa do Estado do Piauí (FAPEPI) pelo apoio financeiro.

\section{REFERÊNCIAS}

1. Noldin, V. F.; Cechinel Filho, V.; Monache, F. D.; Benassi, J. C.; Christmann, I. L.; Pedrosa, R. C.; Yunes, R. A.; Quim. Nova. 2003, 26, 331; Veiga Jr., V. F; Pinto, A. C.; Quim. Nova. 2005, 28, 519.

2. Campêlo, L, M, L.; Almeida, A. A. C.; Freitas, R. L. M.; Cerqueira, G. S.; Sousa, G. F.; Saldanha, G. B.; Feitosa, C. M.; Freitas, R. M.; J. Biomed. Biotechnol. 2011, 678673.

3. Houghton, P. J.; Howes, M. J. R.; Pharmacol. Biochem. Behav. 2003, $75,513$.

4. Bores, G. M.; Huger, F. P.; Petko, W.; Mutlib, A. E.; Camacho, F.; Rush, D.K.; Selk, D. E.; Wolf, V.; Kosley, R. W. Jr.; Davis, L.; Vargas, H. M.; Pharmacol. Exp. Ther. 1996, 277, 728; Barbosa, R. L.; Morais, J. M.; Resck, Z. M. R.; Dázio, E. M. R.; Rev Rene 2012, 13, 1191; Ministério da Saúde (BR). Portaria n. 491/GM, de 23 de setembro de 2010.

5. Minett, T. S. C.; Bertolucci, P. H. F.; Rev. Neurociências 2000, 8, 11.

6. Gupta, A.; Gupta, R.; Phytochemistry 1997, 46, 827; Mukherjee, P. K.; Venkatesan, K.; Malb, M.; Houghton P. J.; Phytomedicine 2007, 14, 289. 
7. Sereniki, A.; Vital, M. A. B. F.; Rev. psiquiatr. Rio Gd. Sul. 2008, 30 (1 Supl.).

8. Yamaguchi, K. K. L.; Alcantara, J. M.; Veiga Junior, V. F.; Acta Amaz. 2012, 42, 541 .

9. Marques, T. H. C.; Santos, P. S.; Melo, C. H. S.; Carvalho, R. B. F.; Lima, L. S.; David, J. M.; David, J. P. L.; Freitas, R. M.; Quim. Nova 2013, 36, 549; Recuero, M.; Vicente, M. C.; Martínez-García, A.; Ramos, M. C.; Carmona-saez, P.; Sastre, I.; Aldudo, J.; Vilella, E.; Frank, A.; Bullido, M. J.; Valdivieso, F.; Aging Cell 2009, 8, 128.

10. Sá, C. G.; Cardoso, K. M. F.; Freitas, R. M.; Feitosa, C. M.; Rev. Ciênc. Farm. Básica Apl. 2012, 33, 211.

11. Rang, H. P.; Dale, M.M.; Ritter, J. M.; Farmacologia, 4a. ed., Editora Guanabara Koogan S.A.: Rio de Janeiro, 2001; Mota, W. M.; Barros, M. L.; Cunha, P. E. L.; Santana, M. V. A.; Stevam, C. S.; Leopoldo, P. T. G.; Fernandes, R. P. M.; Rev. Bras. Pl. Med. 2012, 14, 624; Jung, M.; Park, M.; Molecules 2007, 12, 2130.

12. Gutierrez, C. A. C.; Rev. Colomb. Psiquiatr. 2007, 36, 157; Cunha, U. G. V; Thomaz, D. P.; Marino, C. G.; Balabram, K.; Marquete, C. R.; Geriatria \& Gerontologia 2008, 2, 162; Tiedeman, M.; Kim, E. C.; Flurie, R.; Korch-Black, K.; Brandt, N. J.; Patient Care 2011.

13. Sá, C. G.; Dissertação de mestrado. Universidade Federal do Piauí, Brasil, 2011.

14. Campelo, L. M. L.; Feitosa, C. M.; Tomé, A. R.; Freitas, R. M.; Bol. Latinoam. Caribe Plant. Med. Aromat. 2011, 10, 116; Campelo, L. M. L.; Sá, C. G.; Almeida, A. A. C.; Costa, J. P.; Marques, T. H. C.; Feitosa, C. M.; Saldanha, G. B.; Freitas, R. M.; Pharmazie 2011, 66, 1; Campelo, L. M. L.; Gonçalves, F. C. M.; Feitosa, C. M.; Freitas, R. M.; Pharm. Biol. 2011, 49, 709; Campelo, L. M. L.; De Lima, S. G.; Feitosa, C. M.; Freitas, R. M.; Rev. Bras. Farmacogn. 2011, 21, 668; Gerhardt, C.; Wiest, J. M.; Girolometto, G.; Silva, M. A. S.; Weschenfelder, S.; Braz. J. Food Technol. 2012, 15, 11; Almeida, A. A.; Costa, J. P.; Carvalho, R
B.; Sousa, D. P.; Freitas, R.M.; Brain Res. 2012, 144, 56.

15. Cortez, L. E. R.; Cortez, D. A. G.; Ferreira, A. G.; Vieira, P. C.; Silva, M. F. G. F..; Fernandes, J. B.; Rev. Bras. Farmacogn. 2006, 16, 164; Albarici, T. R.; Vieira, P. C.; Fernandes, J. B.; Silva, M. F. G. F.; Pirani, J. R.; Quim. Nova. 2010, 33, 2130.

16. Melo, M. F. F.; Zickel, C. S.; Acta Bot. Bras. 2004, 18, 73.

17. Kuster R. M.; Rocha L. M.; Cumarinas, cromonas e xantonas. In: Simões C.M.O.; Shenkel, E. P.; Gosmann, G.; Mello, J. C. P.; Mentz L. A.; Petrovick, P. R.; Farmacognosia: da planta ao medicamento. Editora da UFSC: Florianópolis, 2003, 247; Santana, O. A.; Encinas, J. I.; Biotemas 2008, 21, 29.

18. Ellman, G.E.; Coutney, D.; Andres Junior, V.; Featherstone, R.M.; Biochem. Pharmacol. 1961, 7, 88.

19. Rhee, I. K.; Meent, M. V. D.; Ingkanina, K.; Verpoorte, R.; J. Chromatogr. A 2001, 915, 217.

20. Karadsheh, N.; Kussie, P.; Linthicum, D. S.; Toxicol. Lett. 1991, 55, 335.

21. Moyo, M.; Ndhlala, A. R.; Finnie, J. R.; Staden, J. V.; Food Chem. 2010, $123,69$.

22. Lowry, H.; Rosebrough, N. J.; Farr, A. L.; Randall, R. J.; J. Biol. Chem. 1951, 193, 265

23. Liu, R.; Feng, L.; Sun, A.; Kong, L.; J. Chromatogr. A 2004, 1055, 71

24. Trevisan, M. T. S.; Bezerra, M. Z. B.; Santiago, G. M. P.; Feitosa, C. M.; Quim. Nova. 2006, 29, 415.

25. Savelev, S.; Okello E.; Perry, N. S.; Wilkins, R. M.; Perry, E. K.; Pharmacol Biochem Behav. 2003, 3, 661.

26. Kiendrebeogo, M.; Coulibaly, A. Y.; Nebie, R. C. H.; Zeba, B.; Lamien, C. E; Lamien-Meda, A.; Nacoulma, O. G.; Rev. Bras. Farmacogn. 2003, 21,63 .

27. Aremu, A. O.; Amoo, S. O.; Ndhlala, A. R.; Finnie, J. F.; Van Staden, J.; Food Chem Toxicol. 2011, 49, 1122. 